\title{
Making a start on galactoarchaeology?
}

\author{
The discovery last year of Sagittarius, a new dwarf galaxy, prompts a train of speculation about the degree to \\ which the Galaxy has scavenged some of its bulk from the surroundings.
}

How exactly did the Galaxy to which the Sun belongs come into being? These days, of course, everybody knows that a question such as that must be heavily loaded with significance. Did our Galaxy form directly from primordial material (hydrogen, helium and some of the lightest elements) or did it benefit from an earlier recycling through stars, which would have created heavier elements? How long was the gap in time between the beginning of the Universe and the formation of the Galaxy? And given that some of the stars in galactic globular clusters appear to be at least 16 billion years old, how can so many cosmologists sleep easily in their beds at night when they know the latest estimates of Hubble's Constant give an age of the Universe only a little more than half that?

What follows is not, unfortunately, an attempt to answer those philosophical questions in the depth they demand, but rather is a consequence of the guilt evoked by a pile of unread astronomy journals accumulated since last March. Turning them into the library would get them out of the house, and cause no trouble in other ways, but seems a timorous course to follow. Why not browse through them first, to see if they have anything in particular to say. This, then, is the gleaning from half a dozen issues of the Monthly Notices of the Royal Astronomical Society (which now cutely belies its name by appearing every two weeks).

Readers of Nature know, of course, that galaxy formation is one of those central questions for cosmologists, like the meaning of truth for the world at large. Galaxies are supposed to form at points in the expanding Universe where there is an above-average fluctuation of the density of matter, which makes sense. In the days of innocence in cosmology, before 1981, there was a serious difficulty: the primordial density fluctuations would have been so great that only a few very large galaxies would have been formed. But then the inflationary Universe came along, supposing a smoothing expansion of the whole Universe by a factor of $10^{50}$ in the first few hours after the Big Bang, since when the conceptual difficulty has been that of inducing matter to condense into galaxies of any kind. (The famous 'missing mass', responsible for 90 or 95 per cent of the total mass of the Universe, is the other product of inflation.)

It is therefore no surprise that a random set of issues of the Monthly Notices should be richly sprinkled with articles about galaxies and their formation. The place to start is with our own Galaxy, whose most prominent feature is a diskshaped structure some 32,000 parsecs in diameter with the Sun placed roughly half-way from the centre. Who can fail to be intrigued by the case of the dwarf satellite of our own called Sagittarius, discovered only last year and believed then to be in the early stages of tidal disruption by our own (see R. A. Ibata, G. Gilmore \& M. J. Irwin, Nature 370, 194-196; 1995)?

What now emerges is that the disruption of Sagittarius is so great that the authors of the original discovery conclude that it must have made at least one transit of the galactic disk already, in the space of perhaps the past 600 million years. The same argument confirms that Sagittarius is one if the largest of the nine known dwarf galaxies trapped gravitationally by our own; it appears to be comparable in mass with that called Fornax and to be blessed with perhaps 150,000 stars, all of which now seem destined to end up in the galactic bulge. The two Magellanic Clouds, of course, are much larger, but are also showing signs of tidal stress.

The interesting question is not so much about the future as the past. How much of the Galaxy as it is now represents material gathered around the original density fluctuation and how much has subsequently been captured from the surroundings? It is bound to be a messy business, and might even be a fruitless one, to go hunting through the stars of the Galaxy's central bulge, looking for systematic patterns of velocity that would distinguish stars once belonging to nowextinguished dwarf galaxies, but there might be a little luck...

Where, in any case, did the dwarf galaxies come from? The standard explanation is that they were formed from intergalactic clouds either spontaneously or under the stimulus of the Galaxy's own gravitational field, but there is also an irrepressible body of opinion that these aggregations of stars are built around all-purpose black holes. Other schemes would have it that some at least of the dwarf galaxies have been split off from the Magellanic Clouds by tidal interaction with the Galaxy, much as Comet Shoemaker-Levy was broken up into pieces last year by the tidal forces of Jupiter.

Working on the assumption that it should be possible to learn something of these tidal interactions from the distribution of stars in the known dwarf galaxies, Serge Demers (from the University of Montreal) and three colleagues from three different institutions (Mon. Not. roy. Astr. Soc. 274, 491-498; 1995) have carried through a neat statistical analysis of the dwarf galaxies known as Leo I, Leo II, Fornax, Draco and Ursa Minor (all of which, like Sagittarius, are named after 'constellations' by convention).

Their objective is to look for non-random clustering of stars in the dwarf galaxies, using as a statistical criterion the test of whether groups of stars can be linked together by distances no greater than some arbitrary number. The outcome is disappointingly unsurprising; only in Ursa Minor is there a significant departure from randomness, a clump of about 78 stars (out of some 7,000) near the geometrical centre of the galaxy. Dutifully, the authors calculate that the mass of any black hole at the centre would be less than $10^{6}$ solar masses; until more is known of the velocity of the stars in the dwarf galaxy as a whole, the necessity of a black hole to explain this clump is undecidable (and therefore irrelevant).

All this may be intriguing natural history, but it also sustains greater ambitions. Indeed, Ibata and Gilmore (both involved in the discovery of Sagittarius) have heroically collected spectra from 1,500 giant stars in the galactic bulge to construct a model of the dynamics of that region (Mon. Not. roy. Astr. Soc. 275 , 591-604 \& 605-627; 1995). One outcome of that analysis is that the specific angular momentum of the bulge and of the halo that envelops it are essentially the same, but is markedly different from that of the disc. The model-builders will be delighted by all this, but what the rest of us wish to know is how much of the central bulge came with the rest of the Galaxy and how much has been scavenged from elsewhere. Archaeologists would prefer to go looking for remnants of earlier dwarf galaxies, or even for the origin of the globular clusters found predominantly in the parts of the halo near the bulge.

John Maddox 